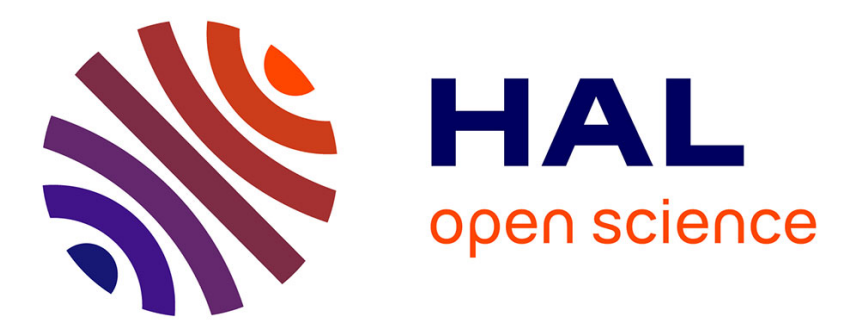

\title{
When More Is Less: Plastic Weakening of Single Crystalline Ag Nanoparticles by the Polycrystalline Au Shell
}

Amit Sharma, Jonathan Amodeo, Nimrod Gazit, Yuanshen Qi, Olivier Thomas, Eugen Rabkin

\section{To cite this version:}

Amit Sharma, Jonathan Amodeo, Nimrod Gazit, Yuanshen Qi, Olivier Thomas, et al.. When More Is Less: Plastic Weakening of Single Crystalline Ag Nanoparticles by the Polycrystalline Au Shell. ACS Nano, 2021, 15 (9), pp.14061-14070. 10.1021/acsnano.1c02976 . hal-03356868

\section{HAL Id: hal-03356868 https://hal.science/hal-03356868}

Submitted on 28 Sep 2021

HAL is a multi-disciplinary open access archive for the deposit and dissemination of scientific research documents, whether they are published or not. The documents may come from teaching and research institutions in France or abroad, or from public or private research centers.
L'archive ouverte pluridisciplinaire HAL, est destinée au dépôt et à la diffusion de documents scientifiques de niveau recherche, publiés ou non, émanant des établissements d'enseignement et de recherche français ou étrangers, des laboratoires publics ou privés. 


\section{When More is Less: Plastic Weakening of Single Crystalline Ag Nanoparticles by the Polycrystalline Au Shell}

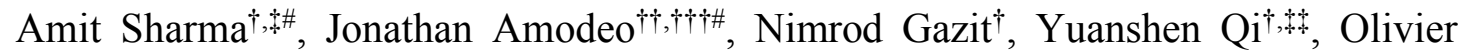
Thomas $^{\dagger \dagger}$, Eugen Rabkin ${ }^{\dagger *}$

†Department of Materials Science and Engineering, Technion - Israel Institute of Technology, 3200003 Haifa, Israel

*Empa, Swiss Federal Laboratories for Materials Science and Technology, Laboratory for Mechanics of Materials and Nanostructures, Feuerwerkerstr. 39, Thun CH-3602,

Switzerland

$\dagger^{\dagger}$ Université de Lyon, CNRS, INSA Lyon, UCBL, MATEIS, UMR5510 CNRS, 69621 Villeurbanne, France

†† Aix Marseille Univ., Université de Toulon, CNRS, IM2NP, Marseille, France

HDepartment of Materials Science and Engineering, Guangdong Technion - Israel Institute of Technology, No.241 University Road, Shantou, Guangdong, P.R. China, 515063

${ }^{\#}$ Equal contributors

*Correspondence to: erabkin@technion.ac.il

\section{ABSTRACT:}

It is well known that in the case of bulk polycrystalline metals, a reduction in the grain size leads to material hardening, since the grain boundaries represent efficient barriers for slip transfer between the adjacent crystalline grains. Here we show that coating single crystalline Ag nanoparticles with a thin polycrystalline Au layer leads to a weakening of the particles. Moreover, while the single crystalline Ag nanoparticles yield in a single large displacement burst when loaded in compression, their Ag-Au core-shell counterparts demonstrate a more homogeneous deformation with signs of strain hardening. Our molecular dynamics simulations demonstrate that particle weakening at low strains is attributed to the plasticity confinement in the polycrystalline shell, in which the grain boundaries play a dual role of dislocations sources and sinks. At higher strains the plasticity within the Ag core is initiated by the dislocations 
nucleating at the Ag-Au interphase boundary. The wide spread of energy barriers for dislocations nucleation at the interphase boundaries and their lower value as compared to the barriers for surface nucleation ensure particle weakening and more homogeneous deformation. The results of this study show that adding imperfect material to superstrong single crystalline metal nanoparticles makes them weaker. At the same time, thin nanocrystalline coatings can be employed to improve the formability of metals at the nanoscale.

KEYWORDS: silver nanoparticles; core-shell nanoparticles; nanoscale plasticity; grain boundaries; dislocation nucleation. 
It is now well-established that plastic deformation of defect-scarce metal samples is controlled by the nucleation of new dislocations. ${ }^{1-5}$ Such samples reach strength levels comparable to the theoretical strength of respective material. Since the bulk metals inevitably contain dislocations and other defects, ${ }^{6}$ the nucleation-controlled plasticity has only been observed in the samples of sub-micrometer dimensions (in the case of uniaxial compression/tension tests), or during nanoindentation of well-annealed single crystals, when the shear stress reaches a maximum in a single point beneath the contact area, and rapidly decays outside. ${ }^{7,8}$ In the nucleation-controlled plasticity regime, the sample deforms elastically up to very high levels of strain in the range of several percent, followed by an abrupt plastic collapse or fracture. For example, the round Ag particles obtained by solid-state dewetting method and compressed along the [111] axis by a flat diamond punch reached the maximum contact pressure of $8 \mathrm{GPa}$ at the onset of plasticity, ${ }^{9}$ while their faceted Au counterparts yielded plastically at the engineering stress values of up to 7-9 $\mathrm{GPa} .{ }^{3}$ For comparison, the yield strengths of pure $\mathrm{Au}$ and $\mathrm{Ag}$ in their bulk form are about 200 and $60 \mathrm{MPa}$, respectively, while the maximum uniaxial compression strength of Au nanopillars prepared by focused ion beam (FIB) milling is about $0.8 \mathrm{GPa} .{ }^{10}$ Introducing defects into the pristine, defect-scarce metal samples leads to a catastrophic drop in their strength: for example, irradiating Au nanoparticles with the $\mathrm{Ga}^{+}$ions in FIB or their plastic pre-straining lead to the decrease of their strength by nearly one order of magnitude. ${ }^{11}$ It should be noted, however, that the ultra-high strength of nanometric samples is both a blessing and a curse, since the new dislocations nucleate in large groups and at random locations, causing dislocation "avalanches" and catastrophic large-strain deformation, which is difficult to control. ${ }^{12}$ Thus, the formability of metals at the nanoscale is one of the central problems in nanomechanics.

In the present work, we address the role played by grain boundaries (GBs) and interphase boundaries in the nucleation-controlled plasticity regime. Several molecular dynamics (MD) studies illustrated the dual role played by the GBs in the nanoscale plasticity: on the one hand, the GBs and their triple junctions represent the sites of heterogeneous nucleation of new dislocations, with the nucleation barrier which is lower than that of homogeneous nucleation in the grain interior, or heterogeneous nucleation on the sample surface. ${ }^{13-15}$ This leads to the GB-induced material softening. On the other hand, the GBs, and especially twin boundaries, are also efficient barriers for gliding dislocation, leading to the nanoscale material hardening. ${ }^{16-18}$ A similar strengthening role is played by the interphase boundaries in metallic nanolayered composite. ${ }^{19,20}$ Finally, in the nanocrystalline samples with the grain size below approximately $10 \mathrm{~nm}$, the GB sliding and other GB diffusion-controlled accommodation processes replace the conventional dislocation-based plasticity. ${ }^{14}$ Also, in the case of nanoparticles and nanowires smaller than $10 \mathrm{~nm}$ in size, the plastic deformation can proceed by a combination of dislocations slip and surface diffusion, ${ }^{21}$ or entirely in the dislocation-less mode, being controlled by the surface self-diffusion, ${ }^{22}$ and diffusion along the punch-particle interface. ${ }^{9}$

The experimental efforts to identify the role of GBs in the nucleation-controlled plasticity have so far been inconclusive. $\mathrm{Ng}$ and Ngan have studied the compressive deformation of bicrystalline $\mathrm{Al}$ micropillars of several micrometers in diameter 
prepared by FIB milling from bulk Al polycrystal. ${ }^{23}$ They found that the presence of GB changes the character of the load-displacement curves, reducing the amplitude of displacement bursts and leading to more continuous "staircase" yielding. Their transmission electron microscopy (TEM) observations revealed high dislocation density in compressed bicrystalline micropillars, much higher than in their single crystalline counterparts tested under identical conditions. It was concluded that the GBs act as dislocation barriers, increasing the dislocation density in the sample and leading to smoother deformation and higher strain-hardening rate. Kunz et al. obtained very different results in their study of bicrystalline Al pillars of smaller diameters ranging from $400 \mathrm{~nm}$ to $2 \mu \mathrm{m} .{ }^{24}$ In contrast to the findings of $\mathrm{Ng}$ and Ngan they observed larger displacement bursts in bicrystalline pillars than in their single crystalline counterparts. Also, the TEM observations revealed the deformation-induced dislocations concentrate in the center of the half-pillar, far from the GB. It was postulated that the GBs serve as dislocation sinks rather than barriers impeding their motion. The difference compared to the behavior observed by $\mathrm{Ng}$ and Ngan was attributed to the difference in the GB geometry and its position in the pillar. Imrich et al. have studied the compression deformation of bicrystalline $\mathrm{Cu}$ pillars of several micrometers in diameter containing a coherent twin boundary (CTB) and a non-special high angle GB (HAGB). ${ }^{25}$ The two bicrystals exhibited very different deformation responses, with the deformation behavior and flow stress of CTB-containing pillar being identical to those of single crystalline pillars of similar diameters, whereas the HAGB-containing pillar demonstrated significantly higher flow stress and strain hardening than its single crystalline counterparts. It was concluded that the investigated HAGB represents an effective barrier to dislocations, while the CTB allows unimpeded cross-slip of dislocations between the two grains. Kheradmand et al. demonstrated a considerable GB strengthening in bicrystalline Ni pillars of about $1 \mu \mathrm{m}$ in diameter, whereas the strength of larger pillars was similar to the strength of their single crystalline counterparts oriented for multiple slip. ${ }^{26}$ Similar trend was also observed during microcompression testing of bicrystalline Fe-2.4 wt.\% Si micropillars: the bicrystalline pillars of $1 \mu \mathrm{m}$ in diameter exhibited higher yield stress than their single crystalline counterparts, while this difference decreased in the pillars of larger diameters. ${ }^{27}$ In all studies described above, the strength of bicrystalline pillars was either comparable to, or higher than the strength of single-crystalline pillars of similar dimensions. ${ }^{19-23}$ However, the conclusions on the effect of GBs on the dislocation nucleation controlled plasticity cannot be drawn from these studies because of abundance of irradiationinduced defects (such as vacancies and small dislocation loops) in the FIB-machined nano- and micropillars. These defects serve as dislocation sources and mask the possible role of GBs in the heterogeneous nucleation of new dislocations. The same can be said about numerous studies in which the FIB-machined pillars made of metal multilayers or nanotwinned metals have been tested. ${ }^{20}$ Recently, it has been shown that the non-equilibrium vacancies are more efficient than CTBs in facilitating the nucleation of dislocations during tensile testing of twinned dislocation-free $\mathrm{Ag}$ nanowires obtained by a wet chemistry method. ${ }^{28}$ 
In the present work, we took advantage of the fact that Ag single crystalline nanoparticles fabricated by solid-state dewetting method exhibit all signs of nucleationcontrolled plasticity when tested in compression. ${ }^{9}$ Metal nanoparticles obtained by solid-state dewetting are equilibrated at high temperatures, hence, they do not contain non-equilibrium vacancies typical for nanostructures obtained by the wet chemistry synthesis, which may mask the effect of GBs on dislocations nucleation. ${ }^{28}$ We coated these defect-free Ag nanoparticles with an ultrathin polycrystalline Au shell which introduced both GBs and interphase boundaries into the system. By performing nanocompression tests on these $\mathrm{Ag}-\mathrm{Au}$ core-shell nanoparticles coupled with $\mathrm{MD}$ simulations, we aim at clarifying the role of grain- and interphase boundaries in the nucleation-controlled plasticity.

\section{RESULTS AND DISCUSSION}

An overall morphology and spatial distribution of Ag nanoparticles fabricated by solidstate dewetting of a $25 \mathrm{~nm}$-thick Ag film deposited on a sapphire substrate is shown in the scanning electron microscopy (SEM) micrograph of Fig. 1a. The particles exhibit hemispherical shape and a wide distribution of sizes, with the diameters ranging from 50 to $700 \mathrm{~nm}$. The morphology of the same nanoparticles after deposition of $15 \mathrm{~nm}$ of $\mathrm{Au}$ is shown in the SEM micrographs in Fig. $1 \mathrm{~b}$ and c. The high-resolution SEM image in Fig. 1c demonstrates the polycrystalline nature of the Au overlayer.

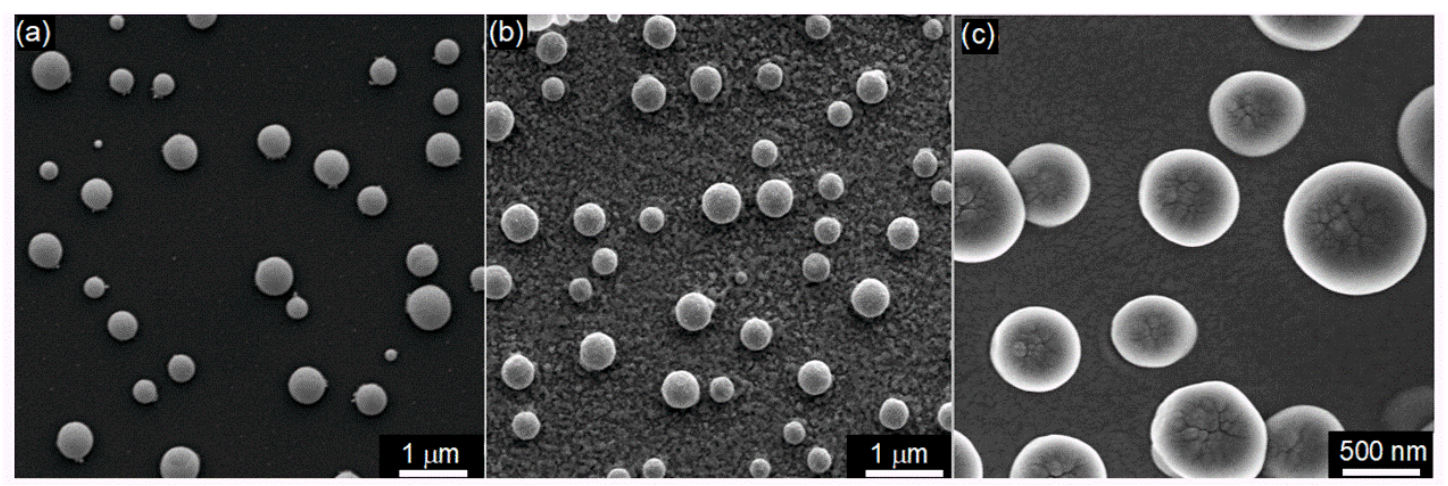

Fig. 1. SEM micrographs of single crystal Ag and Ag-Au core-shell nanoparticles. (a) The SEM micrograph of the as-dewetted Ag film showing hemispherical Ag nanoparticles, $(b, c)$ the same film after depositing of the $15 \mathrm{~nm}$ - thick Au coating. The polycrystalline nature of the Au coating is demonstrated in (c).

The high-angle annular dark-field (HAADF) cross-sectional scanning transmission electron microscopy (STEM) image of an individual Ag-Au core-shell particle is shown in Fig. 2a. It can be seen that the Au coating is thickest at the top of the particle, and its thickness decreases closer to the substrate. This is a result of the shadowing effect during the Au film deposition. The elemental distribution map of the particle acquired with the aid of energy-dispersive X-ray spectroscopy (EDS) in STEM is shown in Fig. $2 \mathrm{~b}$. The varying thickness of the Au shell can be well recognized in this micrograph. Also, a very thin (1-2 nm thick) Ag segregation layer both on the top of the Au shell 
and on the polycrystalline Au film on sapphire in the vicinity of the particle is visible on this elemental map. Moreover, this map confirms that the dark spots at the particlesapphire interface in Fig. 2a correspond to the localized interface pores. Such pores are a result of the Ag outdiffusion along the particle-sapphire interface and formation of the $\mathrm{Au}-\mathrm{Ag}$ solid solution layer on the surface of $\mathrm{Au}$ film (outside of the particle), according to the surface diffusion-induced bulk intermixing mechanism. ${ }^{29}$ The concentration line profile acquired along the dashed black line in Fig. $2 \mathrm{~b}$ in the direction from the particle-sapphire interface to the top of the particle is shown in Fig. 2c. These profiles confirm that no $\mathrm{Au}$ is present in the single crystalline $\mathrm{Ag}$ core, and $\mathrm{Ag}$ forms a thin segregation layer on the top of the $\mathrm{Au}$ shell (see a secondary peak of $\mathrm{Ag}$ concentration at the distance of about $290 \mathrm{~nm}$ from the substrate). No oxygen is present at the Ag-Au interphase boundary (within detection limit of the EDS method). Higherresolution elemental map of the $\mathrm{Au}$ shell region demonstrating that the $\mathrm{Ag}-\mathrm{Au}$ interphase boundary is oxygen-free is shown in Supporting Information (Fig. S1).
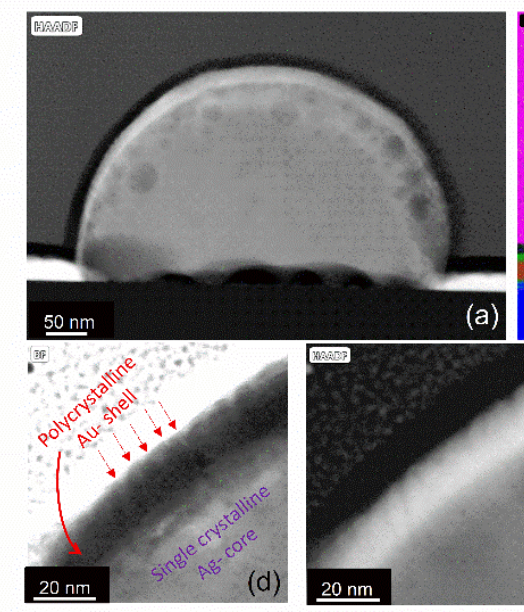

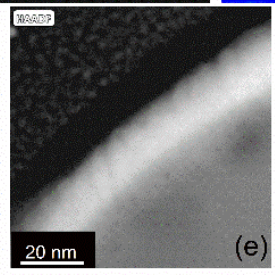

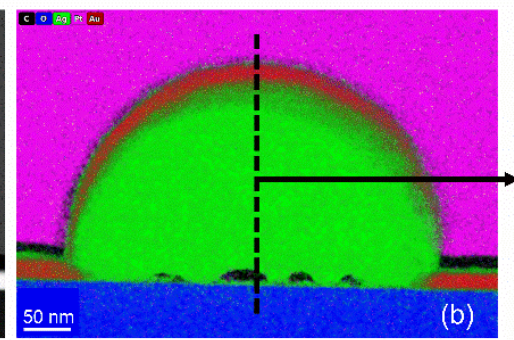

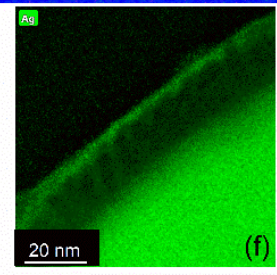

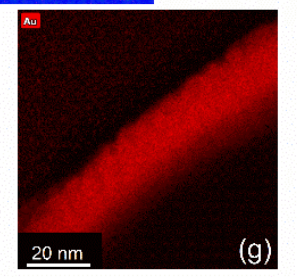
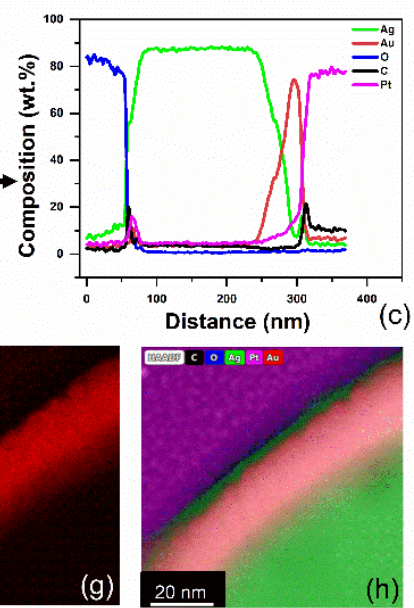

Fig. 2. HAADF-STEM imaging and EDS elemental mapping of core-shell Ag-Au nanoparticles. (a, b) HAADF cross-sectional STEM image of the core-shell Ag-Au nanoparticle, and the corresponding EDS elemental map showing the distribution of $\mathrm{C}, \mathrm{O}, \mathrm{Ag}, \mathrm{Au}$ and $\mathrm{Pt}$ in the particle. (c) Line scans of $\mathrm{C}, \mathrm{O}, \mathrm{Ag}, \mathrm{Au}$ and Pt concentrations acquired along the dashed red line in (b). (d) The BF image of the near-surface region of the particle emphasizing the polycrystalline nature of the Au shell. Several GBs in the shell are marked with the dashed red arrows. (e) The HAADF image of the same region of the particle as shown in (d), demonstrating chemical inhomogeneity of the Au shell. (f, $g$, h) The EDS elemental maps of Ag (f) and $\mathrm{Au}$ (g), and combined EDS elemental maps of $\mathrm{C}, \mathrm{O}, \mathrm{Ag}, \mathrm{Au}$ and $\mathrm{Pt}(\mathrm{h})$.

The bright field (BF) STEM image of a near-surface section of the particle is shown in Fig. 2d. The polycrystalline nature of the $\mathrm{Au}$ shell can be recognized in this micrograph, and several sites where the GBs emerge at the surface are marked with dashed red arrows. The Au shell exhibits a columnar microstructure, with the average diameter of the columnar grains in the range of 10-15 $\mathrm{nm}$. The corresponding HAADF 
image is shown in Fig. 2c, and exemplifies the compositional inhomogeneity of the $\mathrm{Au}$ shell. The corresponding EDS elemental maps of Ag and Au are shown in Figs $2 \mathrm{f}$ and $2 \mathrm{~g}$, respectively; they demonstrate that $\mathrm{Ag}$ is present not only on the surface of the $\mathrm{Au}$ shell but also at the GBs. Finally, the superimposed C, O, Ag, Au and Pt EDS elemental maps are shown in Fig. 2 h.

The tilted SEM images of the Ag-Au core shell nanoparticles before and after the in-situ micro-compression test in SEM are shown in Fig. 3a and b, respectively. These micrographs demonstrate that plasticity is localized at the top of the particle, with only minor increase of the particle dimensions outside the compressed region. Also, these images demonstrate that only one particle was compressed during the test. The shape of the deformed Ag-Au particle does not show any sign of catastrophic plastic flow, which is in strong contrast with the pancake-like shapes of the compressed singlecrystalline Ag particles (see Fig. 3c and d), and also Au and Ni particles. ${ }^{3,4}$

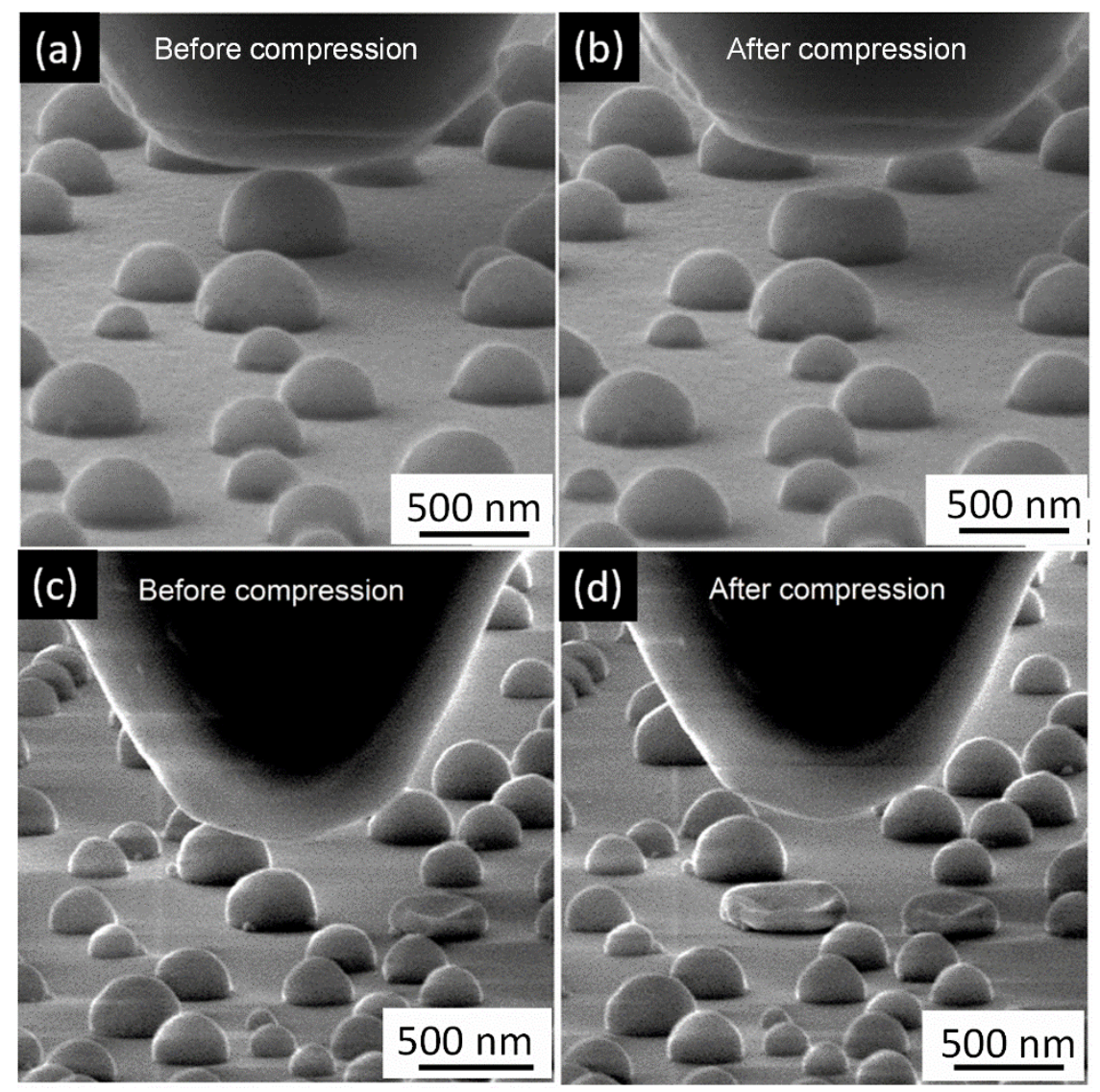

Fig. 3. SEM imaging of Ag-Au and Ag nanoparticles compression tests. (a, b) AgAu core-shell nanoparticles before (a) and after (b) test. (c, d) Single crystalline Ag nanoparticles before (c) and after (d) test.

The load-displacement curves of the Ag-Au core-shell nanoparticles with the diameters of 320-515 nm obtained during in-situ compression tests are shown in Fig. 4a, together with several typical load-displacement curves of pristine (as-dewetted) Ag particles shown for comparison (based on the data of Sharma et al.). ${ }^{9}$ The loaddisplacement curves of pristine Ag nanoparticles exhibit typical features of plastic 
deformation of pristine metal structures controlled by dislocation nucleation, i.e. a Hertzian-like elastic deformation up to very high values of displacement, followed by a large displacement burst. This burst is related to simultaneous nucleation of a large number of dislocations resulting in a sudden drop of particle strength. This massive nucleation event is so fast that the electronic feedback loop system of the PicoIndenter responsible for maintaining the constant displacement rate does not manage to react, and the punch rams into the particle, resulting in flattened particle at the end of the test (see Fig. 3d). For the sake of clarity, the load-displacement curves of pristine Ag nanoparticles are shown separately in Fig. S2 of the Supporting Information. The loaddisplacement curves of the Ag-Au core-shell nanoparticles exhibit a very different behavior from that of single-crystalline Ag nanoparticles. Indeed, here plasticity sets on at very early stages of deformation, and the large singular displacement bursts are lacking. The core-shell particles deform in a more gradual manner, with a large number of small displacement bursts separated by short elastic loading sections. This type of load-displacement curve is sometimes referred to as a "staircase yielding", and it is related to the operation of numerous surface or internal dislocation sources. ${ }^{8,30}$ Overall, the deformation of the core-shell nanoparticles can be much better controlled than that of their single crystal Ag counterparts. Also, no change of slope or any other singularity is observed in the range of depths between 15 and $40 \mathrm{~nm}$, corresponding to the sum of the thickness of the Au shell and of the maximum displacement of single crystalline Ag nanoparticle. Still, a large load drop at the displacement of about $50 \mathrm{~nm}$ on the loading curve of the core-shell nanoparticle of $515 \mathrm{~nm}$ in diameter may be associated with a multiple and simultaneous dislocations nucleation in the Ag core, albeit at a smaller scale as compared to single crystalline Ag nanoparticles, so that the feedback loop system of the instrument manages to capture this event. To provide a more detailed view of the initial part of the load-displacement curves of the core-shell nanoparticles, a sub-set of these curves acquired with the maximum displacement below $40 \mathrm{~nm}$ is shown in Fig. 4b. Both Figs $4 a$ and $4 b$ demonstrate that the effective slope of the loaddisplacement curves of the core-shell nanoparticles is much shallower than that of the pristine Ag particles of comparable diameters in the elastic deformation region. Therefore, one can characterize the observed deformation response of the two types of particles as "plastic weakening". To provide a quantitative measure of "weakness" of the Ag-Au core-shell nanoparticles, we split several selected nanoparticles in Ag/Ag$\mathrm{Au}$ pairs, such that in each pair the diameter of the core-shell particle is by $20-30 \mathrm{~nm}$ larger than the diameter of its single crystalline counterpart (this difference approximately corresponds to the Au shell of $15 \mathrm{~nm}$ in thickness). We then compared the maximum load at the onset of displacement burst for the single crystalline nanoparticle with the load on its core-shell counterpart at the same displacement. The results of this comparison (see Table 1) show that the single crystalline nanoparticles are by a factor of two to four stronger than their larger core-shell counterparts. It should be noted that according to Hertz's contact model, for equal elastic displacements the load on the larger particle should be larger. The Table 1 demonstrates an opposite trend, clearly indicating that plasticity in the core-shell nanoparticles sets on at much lower loads than in their single crystalline counterparts. It should be noted that determining 
the yield stress of the core-shell nanoparticles is problematic because the plasticity sets on at very low loads approaching the load noise level of the instrument. Thus, counterintuitively, adding material in the form of ultrathin polycrystalline coating to the pristine single crystalline Ag particles leads to the plastic weakening of the latter. This is even more puzzling considering that the deposited material contains numerous HAGBs and adds a Ag-Au interphase boundary to the system, which, following the analogy with bulk materials and metallic multilayers, should contribute to material strengthening.

(a)

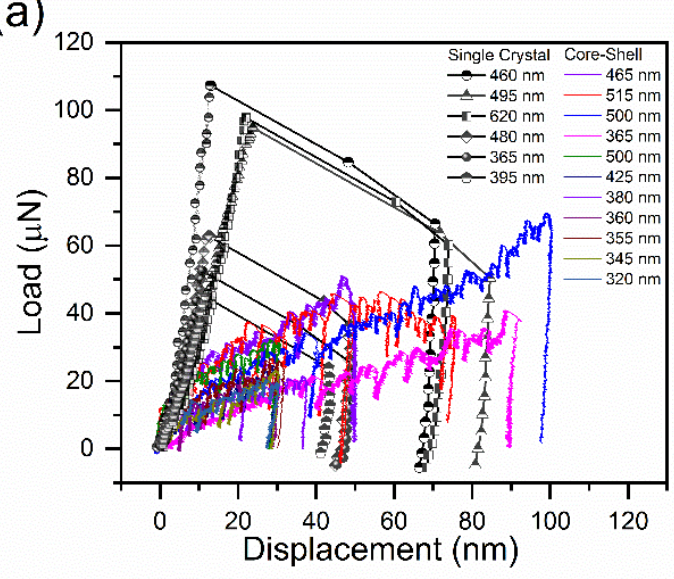

(b)

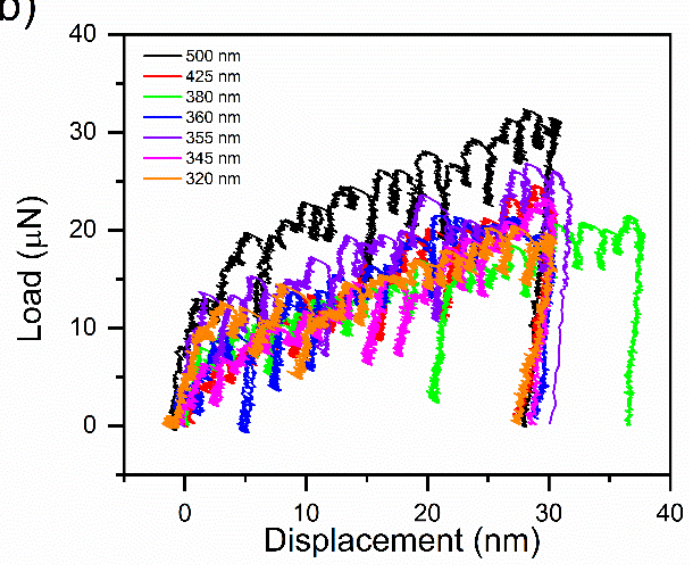

Fig. 4. Load-displacement curves of the studied nanoparticles. (a) The loaddisplacement curves of the Ag-Au core-shell nanoparticles, shown together with several representative loading curves of pristine (as-dewetted) single crystalline Ag particles (adapted from Sharma et al. ${ }^{9}$ with permission from Wiley-VCH); (b) the load-displacement curves of Ag-Au core-shell nanoparticles with the maximum displacement below $40 \mathrm{~nm}$.

Table 1. Six pairs of single crystalline and core-shell nanoparticles selected in a way that the diameter of core-shell nanoparticles is by $20-30 \mathrm{~nm}$ larger than that of its single crystalline counterpart (this difference approximately corresponds to the thickness of polycrystalline Au shell). A comparison of the maximum load on the Ag single crystalline nanoparticle with the load on its core-shell counterpart in the pair at the same displacement demonstrates that the core-shell nanoparticles are by a factor of two to four weaker than their single crystalline counterparts. 


\begin{tabular}{cccccc}
\hline \multirow{2}{*}{ No. } & Displacement $(\mathrm{nm})$ & \multicolumn{2}{c}{ Single Crystalline Ag particles } & \multicolumn{2}{c}{ Core-shell Particles } \\
& Diameter $(\mathrm{nm})$ & Load $(\mu \mathrm{N})$ & Diameter $(\mathrm{nm})$ & Load $(\mu \mathrm{N})$ \\
\hline 1 & 13 & 320 & 27 & 345 & 14 \\
\hline 2 & 25 & 360 & 83 & 380 & 16 \\
\hline 3 & 12 & 395 & 44 & 425 & 12 \\
\hline 4 & 13 & 460 & 90 & 485 & 23 \\
\hline 5 & 12 & 480 & 62 & 500 & 21 \\
\hline 6 & 22 & 495 & 94 & 515 & 30 \\
\hline
\end{tabular}

In order to understand the striking difference in nanomechanical behavior of the $\mathrm{Ag}$-Au core-shell nanoparticle and its single crystalline counterpart, the microstructure of the former has to be analyzed in detail.

The molecular beam epitaxy (MBE) deposition of Ag on Au nanowhiskers under ultra-high vacuum conditions resulted in heteroepitaxial coating. ${ }^{31}$ In the present work, there was a vacuum break between the dewetting heat treatment of the Ag film and the deposition of Au coating. This resulted in absorption of gases from the ambient and prevented formation of heteroepitaxial Au coating on the Ag particles. Thus, the growth of the Au coating has probably proceeded in the Volmer-Weber mode, ${ }^{32}$ resulting in a columnar polycrystalline microstructure. The enrichment of the GBs in the Au shell with Ag, and the presence of Ag-rich layer on the surface of the Au shell clearly indicate that $\mathrm{Ag}$ diffused along the GBs in Au at room temperature. Since bulk interdiffusion in the Ag-Au system at room temperature is frozen out, this means that the GBs in the $\mathrm{Au}$ shell represent the fast diffusion paths. This, in turn, indicates that these GBs are of the random high angle type (HAGBs), since the diffusion along the low-angle GBs and the CTBs is not much different from that in the bulk. ${ }^{33} \mathrm{~A}$ detailed estimate presented in Supporting Information indicates that that the average diffusion distance of $\mathrm{Ag}$ atoms along the HAGBs in the Au shell after $24 \mathrm{~h}$ of storing at room temperature is 100-300 $\mathrm{nm}$, much larger than the thickness of the Au shell. This enables the formation of Agenriched layer on the surface of the shell.

Let us now consider two possible reasons for the early plastic yielding of the AgAu core-shell nanoparticles:

(1) The polycrystalline Au shell is characterized by an increased surface roughness due to the GB grooving phenomenon. ${ }^{34}$ Could it lead to particle weakening? Indeed, the characteristic GB grooves on the surface of the Au shell can be recognized in the BFSTEM micrograph of Fig. 2d. This leads to the hemispherical morphology of the individual grains in the Au shell, resulting in much higher local stress concentration in the contact zone as compared to the indentation geometry of pristine Ag nanoparticles. However, once the diamond punch reached the roots of the GB grooves the gaps between the multiple contact zone become closed, and the overall contact geometry of the core-shell nanoparticle becomes similar to that of its pristine Ag counterpart. A simple estimate based on Hertz's contact theory (see Supporting Information) yields 
the following result for the maximum mean contact pressure, $p_{m}$, achieved at the moment the punch reaches the roots of GB grooves:

$$
p_{m}=\frac{4 E^{*}}{3 \pi}\left[1-\left(1-\left(\frac{\gamma_{b}}{2 \gamma_{s}}\right)^{2}\right)^{1 / 2}\right]
$$

where $\gamma_{\mathrm{b}}$ and $\gamma_{\mathrm{s}}$ are the HAGB energy and the surface energy, respectively, of Au (we neglect the presence of Ag segregation layer because the surface and GB energies of $\mathrm{Ag}$ are close to those of $\mathrm{Au}$ ). The parameter $E^{*}$ represents the effective elastic modulus of the system "gold surface-diamond punch", yet for our rough estimates, we will neglect the elastic deformation of diamond and approximate $E^{*}$ by the isotropic Young modulus of $\mathrm{Au}, E^{*} \approx 79 \mathrm{GPa}$. For the random HAGB in $\mathrm{Au}, \frac{\gamma_{b}}{2 \gamma_{s}} \approx 0.15,{ }^{35}$ yielding $p_{m} \approx 3.6 \mathrm{GPa}$. Though this high mean contact pressure can cause the nucleation of dislocations due to the stochastic nature of the latter, it is significantly lower than the values of mean contact pressure of about $8 \mathrm{GPa}$ at the onset of plastic yielding which were measured in pristine Ag nanoparticles. ${ }^{9}$ Since the early staircase plastic yielding was observed in all studied core-shell nanoparticles, we conclude that the GBassociated surface roughness cannot explain this kind of weakening.

(2) Could a decrease of dislocations nucleation energy barriers at the HAGBs in the $\mathrm{Au}$ shell and/or at the Ag-Au interphase boundary lead to particle weakening? As such question can only be answered at the atomistic level, we performed MD compression simulations of the single crystal $\mathrm{Ag}$ and $\mathrm{Ag}$ - $\mathrm{Au}$ core-shell nanoparticles, closely mimicking the microstructure of the particles studied in the experiment. Ag-Au coreshell nanoparticles with diameter $d$ of up to $20.1 \mathrm{~nm}$ were designed using a Voronoi tessellation with random grain orientation for the shell grains surrounding a $<111>$ oriented hemispheric Ag single crystal (see Figure 5). Various Voronoi grain sizes $g$ and aspect ratios $\rho=r_{A g} / r$ ( $r_{A g}$ and $r$ being the Ag core and the full nanoparticle radii, respectively) were investigated. Figure $5 \mathrm{~d}$ shows the relaxation of a core-shell structure during the computational "fabrication" process. It was found that for $g=6 \mathrm{~nm}, \rho=0.8$ and an equilibration temperature of $150 \mathrm{~K}$ the relaxation of both the Ag-Au core-shell interface and the $\mathrm{Au}$ shell GBs proceeds without a major recrystallization process in the shell and without dislocation nucleation in the Ag core, leading to a stable structure over time. 


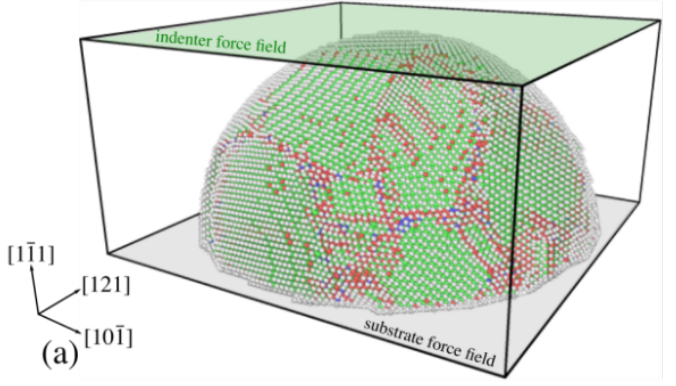

(b)
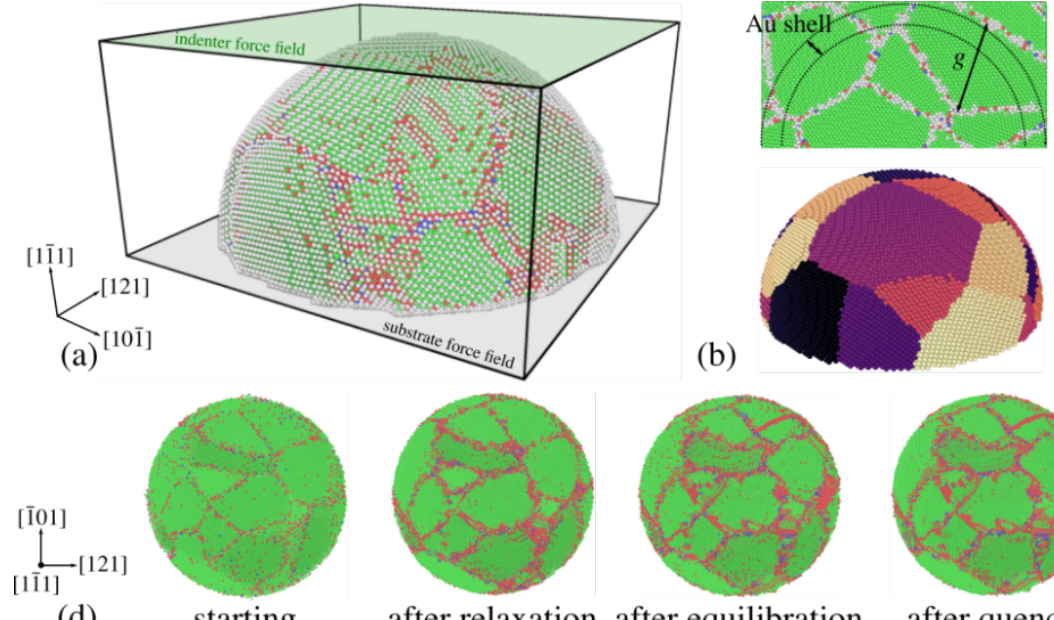

(d)

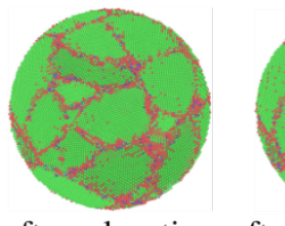

after relaxation after equilibration

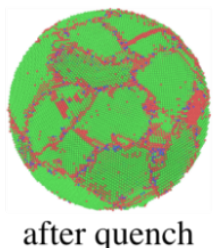

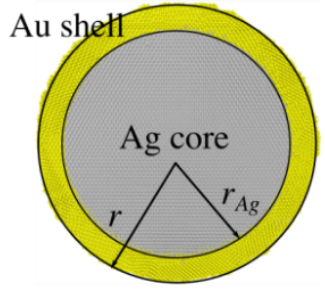

(c)

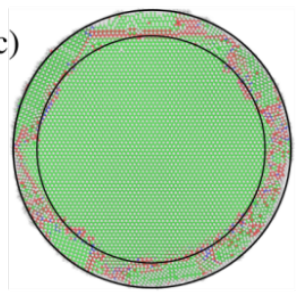

Fig. 5 Simulations setup and equilibration of $\mathrm{Ag}$-Au hemispherical core-shell nanoparticles. (a) Ag-Au core-shell nanoparticle compression setup, (b) Carving of a polycrystalline shell out of a Voronoi polycrystal. $g$ refers to the characteristic grain size of the original polycrystal. In the bottom image, the color of the atoms scales with the grain index, (c) Bottom views of an Ag-Au core-shell nanoparticle. In the top image, atoms colors refer to atomic species while local atomic environment is shown in the bottom figure, (d) Top view of the shell grain structure evolution during fabrication, including the 'starting' configuration: just as-carved from a larger block, 'after relaxation': after molecular statics energy minimization, 'after equilibration': heating to the temperature of $T=150 \mathrm{~K}$ and equilibration for $1 \mathrm{~ns}$ in the NVT ensemble, and 'after quenching'. Atoms in a perfect crystal (fcc) environment are colored in green, atoms in GBs are in red, blue and light-grey (respectively hcp, bcc and other non-fcc local environments). Surface atoms are colored in light-grey. For the sake of clarity, they are removed from (d).

Figure 6 shows the MD load - displacement curves for a pure Ag single crystal and five $\mathrm{Ag}-\mathrm{Au}$ core-shell nanoparticles of the same dimension but exhibiting various shell microstructures (labelled s1 to s5), deformed up to $20 \%$ engineering strain. The Ag load - displacement curve shown in Figure 6a is typical of MD nanoparticle compression tests. It starts with a linear elastic regime up to a displacement of $2.75 \AA$ (equivalent to a strain of $\varepsilon \approx 2.78 \%$ ), where a first load relaxation is observed. Here we use the loadcontrol model proposed by Mordehai et al. ${ }^{3}$ to better compare displacement-control MD simulation outcome to the experiments. Indeed, while the experimental compression tests were performed using the displacement-control mode, the fast nucleation of multiple dislocations and the following strain burst are more typical for the load-control mode, simply because the feedback loop system of the PicoIndenter does not have time to respond. In the simulation, the first load drop is associated to the nucleation of $1 / 6<112>\{111\}$ Shockley partial dislocations from the surface (Figure $7 a)$ that quickly escape the sample, followed by a renewed elastic loading of the particle. This process repeats during the whole simulation and is accompanied by the storage of extended stacking faults inside the sample. This deformation process is known to be size- 
dependent, and is quite comparable to the plastic bursts observed in the experimental compression tests of single crystalline Ag nanoparticles. Note that no nanotwins were observed in the course of deformation.

The load-displacement curves of the Ag-Au core-shell nanoparticles shown in Figure $6 \mathrm{~b}$ are strikingly different from those of their single crystalline Ag counterparts. Firstly, the initial stages of deformation of the Ag-Au core-shell nanoparticles proceed in pseudo-elastic regime, with the slope of load-displacement curves being much shallower than the corresponding slope of the Ag single crystal nanoparticles deforming in the elastic regime. Here, the slopes of the load - displacement curves vary broadly, which is associated with both the contact and the shell microstructure below the indenter. The pseudo-elastic regime is characterized by stress-induced local rearrangements localized below the indenter, in the Au shell (see e.g., Figure 7b). Contrary to what is usually observed in MD compression tests of single crystalline nanoparticles (see Refs. ${ }^{3,36}$ for examples), here the pseudo-elastic regime does not end at a deterministic critical strain, i.e. the critical strain spreads from 2.8 to $7.1 \%$ (see the set of downward arrows for displacements lower than $8 \AA$ in Figure $6 b$ ).

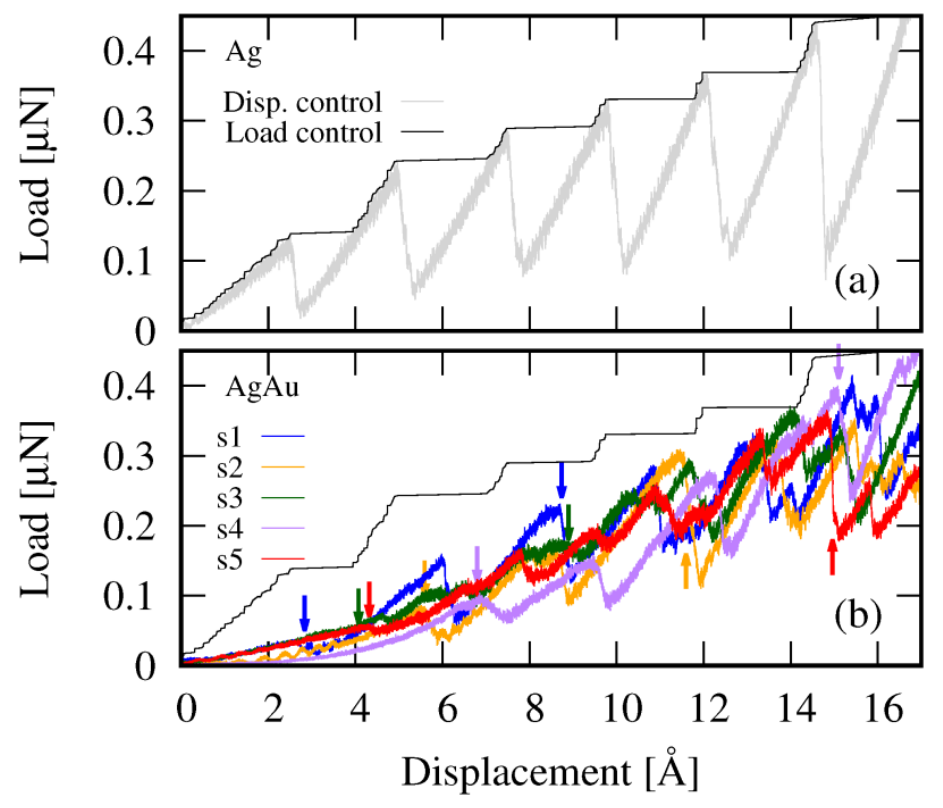

Fig. 6 Load - displacement curves obtained by MD nanocompression simulations (a) Ag single-crystal nanoparticle $(d=20.1 \mathrm{~nm})$. The light-grey curve refers to MD raw data while the black curve is the load-control envelope retrieved using Mordehai et al. method. $^{3}$ (b) Ag-Au core-shell nanoparticles $(d=20.1 \mathrm{~nm})$. The first dislocation nucleation events in the nanoparticle shell and later in the core are marked with the arrows (for displacements smaller and larger than $8 \AA$, respectively). The load-control mechanical response of single crystal Ag particle (black curve) is shown for comparison.

The end of the pseudo-elastic regime is characterized by the nucleation of Shockley partial (leading and often consecutive trailing) dislocations from the surface of shell grains that are in direct contact with the indenter. As shown Figure 6b, this process 
happens at a lower load than in the Ag single crystal which confirms the crucial role of the shell microstructure. Then, plasticity proceeds in two consecutive steps. In a first step, dislocations nucleate and propagate only in the shell grains; they are further absorbed in $\mathrm{Au}$ shell GBs or at the Ag-Au interphase boundary. This process is illustrated in Figure 7c (s2, at a displacement of $6.2 \AA$ ), where a freshly nucleated stacking fault passing through a shell grain is emphasized using the atomic displacement analysis. This process is characterized by load drops of lower amplitude, when compared to those of the single-crystalline Ag nanoparticle, which makes identifying the yield point of the core-shell nanoparticle difficult. This deformation regime is characterized by an overall strain-hardening behavior. The nucleation/propagation events in the shell persist up to the strains of several percent (arrows in Figure $6 \mathrm{~b}$ for displacements larger than $8 \AA$ ) where, in a second step, dislocations start to propagate into the Ag nanoparticle core. An example of this process is illustrated Figure $7 \mathrm{c}$ in the s 2 case where, at a displacement of $11.7 \AA$, two correlated Shockley partial dislocations propagate inside the core. At $11.8 \AA$ displacement, several stacking faults that extend from the core-shell interface of $s 2$ down to the substrate (Figure 7c) are easily identifiable, confirming the plastic deformation of the entire nanoparticle. Note that these subsequent nucleation events in which the nanoparticle core is involved are characterized by load drops of larger amplitude when compared to plastic deformation of the shell, in agreement with the experimental observations.

A detailed analysis of the MD simulations confirms the multiplicity of the elementary deformation processes that occur during this second step of deformation. Indeed, we have observed dislocations simultaneously emitted from both the shell surface and the Au-Ag interphase boundary (see supplementary Movie M1 in Supporting Information). For example, dislocation loops that were originally nucleated in the shell grains can be stored in the shell for some time (either pinned by GBs or by other extended defects), and then reach the interphase boundary after some atomic rearrangements. Afterwards, the interphase boundary can undergo an instantaneous shear (observed for both coherent and incoherent Au-Ag interphase boundaries), reemit the dislocation into the Ag core right after absorbing shell dislocation, or emit the dislocation into the $\mathrm{Ag}$ core during spontaneous, time-uncorrelated, nucleation events.

It should be noted that these second step nucleation events occur at a lower load than surface dislocation nucleation within the single crystalline Ag particle at comparable value of indenter displacement (Figure 6b). This means that, overall, the energy barriers for dislocation nucleation, especially those at the Ag-Au interphase boundary, are lower than that on the surface of the single crystalline Ag particle. 


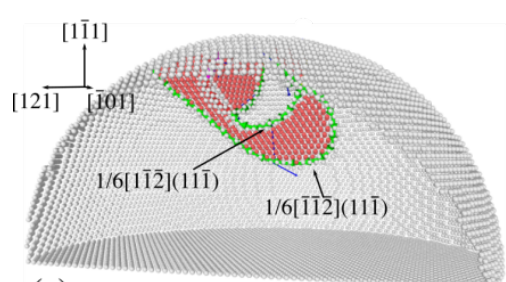

(a)

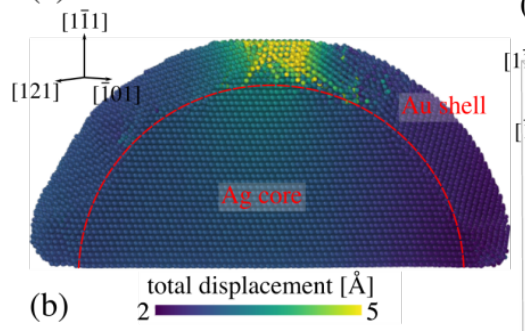

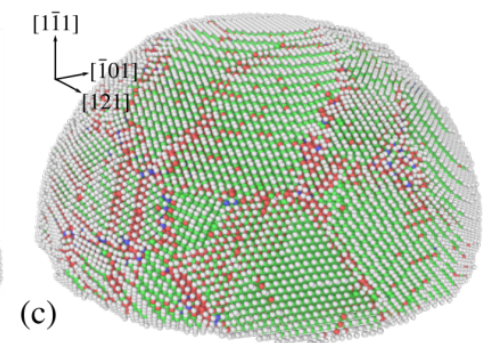

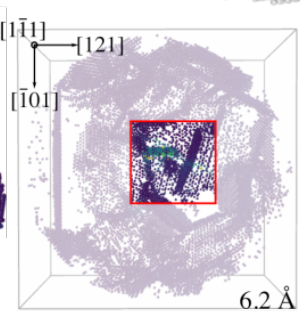

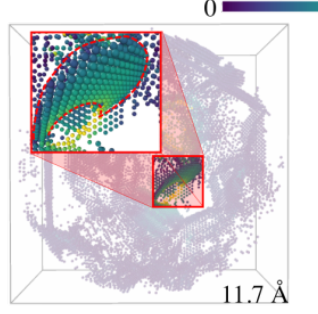

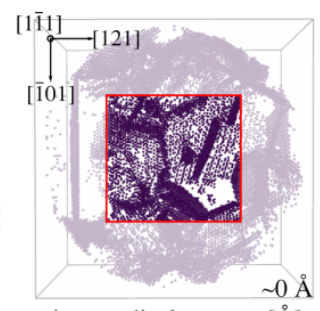

lacement $[\AA]$

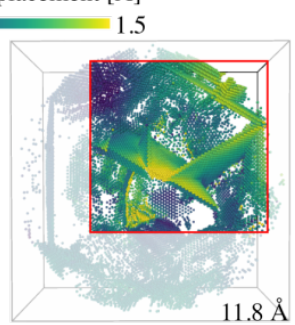

Fig. 7 MD simulation of Ag single-crystalline and Ag-Au core-shell nanoparticles. (a) Nucleation of $1 / 2<110>\{111\}$ dislocation in Ag nanoparticle at a displacement of $2.75 \AA$. Atoms colored in light-grey refer to surfaces. Green curves, red atoms and blue arrows refer to partial dislocations, stacking faults and Burgers vectors, respectively. Perfect crystal atoms are removed for the sake of clarity. (b) Cross-section of an Ag-Au core-shell nanoparticle (s4) deformed in the pseudo-elastic regime (displacement of $5.7 \AA$ ). Atoms colors show the total atomic displacements (with starting configuration serving as reference). (c) Dislocation nucleation in Ag-Au core-shell nanoparticle (s2) including the starting configuration with atom colored using local structure environment (green $=$ fcc, blue $=$ bcc, red $=$ hcp, white $=$ others) and bottom views of the nanoparticle recorded at displacements of about $\sim 0$ (recorded after $20 \mathrm{ps}$ of similations), 6.2, 11.7 and $11.8 \AA$ with atoms colored using instant atomic displacements (with the configuration captured 20 ps earlier serving as reference).

The shell-localized deformation observed in our MD simulations enables to understand the striking difference in the mechanical behavior of the single crystalline $\mathrm{Ag}$ nanoparticles and their Ag-Au core-shell counterparts observed experimentally. Indeed, the localized plastic events of small amplitude in the Au shell impede the massive singular dislocations nucleation event in the Ag core responsible for the catastrophic shape change. These localized plastic events occur at lower loads and take more time, enabling an adequate response of the electronic feedback loop system of the PicoIndenter. This is why the experimental load-displacement curves in Fig. 4 exhibit load drops which are typical for the displacement-control compression mode.

Furthermore, at the moment dislocation nucleation or transmission in the Ag core begins, the highly deformed shell ensures relatively large contact area between the punch and the particle encompassing many $\mathrm{Au}$ grains. The Ag-Au interphase boundaries between the highly deformed Au shell and Ag core are characterized by different macroscopic geometrical degrees of freedom (i.e. relative orientations of the $\mathrm{Au}$ grains and $\mathrm{Ag}$ particle) and, hence, different energy barriers for dislocation 
nucleation. This wide spread of nucleation barriers ensures that only few dislocations are nucleated at a time at the interphase boundaries with the lowest nucleation barrier. Thus, the gradual deformation which was localized in the Au shell continues in the same manner after the plasticity spreads into the $\mathrm{Ag}$ core. This is different from the plasticity of single crystalline Ag particle: because of the axial symmetry of the system all potential surface nucleation sites exhibit the same nucleation barrier, leading to the dislocations avalanche after the first nucleation event and concomitant displacement burst. Overall, the localized plasticity in the polycrystalline shell and the following limited plasticity in the Ag core enable a discretized, more homogeneous, and better controlled compression experiment, in contrast to the massive strain burst characteristic of the single crystal nanoparticle. The core-shell nanoparticles can be plastically deformed to any pre-determined value of strain, largely retaining their axial symmetry (see Fig. 3b), contrary to their single crystalline counterparts which instantly collapse to a distorted pancake shape once the critical stress for the dislocations nucleation is reached (see Fig. 3d). However, this ability of controlled plastic deformation of the core-shell nanoparticles comes at a price, as their overall strength is lower than that of single crystalline nanoparticles.

\section{CONCLUSION}

In conclusion, we have demonstrated that coating the hemispherical single crystalline Ag nanoparticles with a thin polycrystalline layer of Au significantly reduces their compressive strength, in spite of the fact that the coating thickness is less than ten percent of the particle height. Also, the deformation mode of the particles changes from the single large displacement burst leading to a catastrophic plastic collapse of the single crystalline particles to a more gradual plastic deformation with moderate load drops in the case of Ag-Au core-shell particles. Thus, the core-shell particles are weaker than their single crystalline counterparts, but their plastic deformation can be better controlled. The atomistic molecular dynamics simulations of the single crystalline $\mathrm{Ag}$ nanoparticles and of the Ag-Au core-shell nanoparticles with a single crystalline core and polycrystalline shell have revealed the atomistic mechanisms of plastic deformation of the particles. In both cases, the deformation was controlled by nucleation of new dislocations, however, in the core-shell nanoparticles, the grain and interphase boundaries served both as sources and sinks of dislocations, leading to plasticity confinement in the shell at low strains and to the particle weakening. The plasticity of the Ag core was then initiated by dislocation nucleation or transmission at the Ag-Au interphase boundary. We proposed that plastic weakening of the core-shell nanoparticles observed in the experiment is associated with the variations of atomic structure of the $\mathrm{Au} \mathrm{GBs}$ and $\mathrm{Ag}-\mathrm{Au}$ interphase boundaries, with the resulting wide spread of the energy barriers for dislocations nucleation. The results of this work can be utilized for solving one of the main problems of nanomechanics - the difficulty of controlling the final shape during the plastic forming caused by large strain bursts. ${ }^{12}$ Our results indicate that thin nanocrystalline coatings can make the deformation more homogeneous and easier to control, leading at the same time to plastic weakening. 


\section{METHODS}

Fabrication of Ag and core-shell Au-Ag nanoparticles

The experimental details of the preparation of Ag-Au core-shell nanoparticles are presented in the work of Gazit et al. ${ }^{29}$ In short, after deposition of the $25 \mathrm{~nm}$ thick $\mathrm{Ag}$ film on the polished and cleaned $c$-oriented sapphire substrate (by thermal evaporation, VINCI E-PVD4 deposition system), the sample was annealed in the ambient air at the temperature of $700{ }^{\circ} \mathrm{C}$ for $5 \mathrm{~h}$. This annealing resulted in solid-state dewetting of the $\mathrm{Ag}$ film and the formation of isolated hemispherical single-crystalline $\mathrm{Ag}$ particles. Afterward, a $15 \mathrm{~nm}$-thick Au coating was deposited on the sample with Ag particles employing electron beam deposition in the Airco Temescal FC 1800 system. The deposition was performed at room temperature.

\section{$\underline{\text { Characterization of nanoparticles }}$}

The morphology of Ag and Ag-Au core-shell nanoparticles was characterized in the high-resolution scanning electron microscope (Carl Zeiss Ultra Plus) using a secondary electron detector, at an acceleration voltage of $4 \mathrm{keV}$. The microstructure and composition analysis of the core-shell particle was performed in a high-resolution transmission electron microscope (HRTEM; FEI 80-300 Themis $\mathrm{G}^{2}$ ) using an accelerating voltage of $300 \mathrm{keV}$. The measurements were performed in STEM mode using a high-angle annular dark-field (HAADF) and bright field (BF) detectors. Energy-dispersive X-ray spectroscopy (EDS) maps were acquired by a Dual-X detector (Bruker) which has an effective solid angle of $1.76 \mathrm{sr}$. The cross-section TEM lamella was prepared in a dual-beam focused-ion beam (FIB)-SEM microscope (FEI Helios NanoLab DualBeam $\mathrm{G}^{3} \mathrm{UC}$ ).

\section{$\underline{\text { In-situ nanomechanical testing }}$}

The in-situ nanomechanical compression tests of particles were performed in a scanning electron microscope using a Hysitron PI85 PicoIndenter equipped with a flat diamond punch tip with a projected diameter of $1 \mu \mathrm{m}$. All the particles were tested in a displacement-controlled mode with a constant nominal displacement rate of $1 \mathrm{~nm} \mathrm{~s}^{-1}$. For better accuracy, the load-displacement data were recorded after the thermal drift became less than $0.002 \mathrm{~nm} \mathrm{~s}^{-1}$. The secondary electron images were collected before and after the compression to confirm that a single particle is deformed at a time.

In-silico fabrication of nanoparticles

$\mathrm{Ag}$ and $\mathrm{Ag}-\mathrm{Au}$ nanoparticles were designed using the Atomsk tool ${ }^{37}$ combined to homemade subroutines. First, an Ag hemispheric single crystal with radius $r_{A g}$ is carved out from a larger block, with the basis normal oriented along $<111>$. This as-carved crystal is later used either as a nanoparticle itself, or as the core of a Ag-Au core-shell particle. Then, an Au shell is sliced out from a Voronoi polycrystalline block of atoms and joined to the surface of the Ag core. The original polycrystalline block is made of randomly oriented grains with mean grain size $g$ that scales with the final grain size of 
the shell. In this study, $g$ ranges from 6 to $12 \mathrm{~nm}$. Diameters for Ag single crystal and $\mathrm{Ag}$-Au core-shell nanoparticles range up to $d=20.1 \mathrm{~nm}$, and the relative amount of materials $\mathrm{Ag} /(\mathrm{Ag}+\mathrm{Au})$ is characterized by parameter $\rho=r_{A g} / r$, where $r$ is the total (external) radius of the nanoparticle.

After fabrication, the sample is equilibrated using the LAMMPS code and the Zhou et al. embedded atom method (EAM) potential for metallic alloys. ${ }^{38,39}$ Among others, the Zhou potential was successfully used to model metals multilayer deposition, ${ }^{40}$ contact between $\mathrm{Ag}$ and Au nanowires, ${ }^{41}$ and core-shell nanoparticles. ${ }^{42}$ We verified its transferability to the mechanical behavior of $\mathrm{Ag}$ and $\mathrm{Au}$ by verifying a set of relevant material properties, including elastic constants, stacking fault and surface energies (see Supporting Information). After a preliminary minimization run down to a force norm of $10^{-6} \mathrm{eV} / \AA$ using the FIRE algorithm, ${ }^{43}$ atomic velocities are set and the sample is quickly heated up to a $150 \mathrm{~K}$ temperature plateau maintained during $1 \mathrm{~ns}$ using NVT $\mathrm{MD}$ and the Nosé-Hoover thermostat. During equilibration at $T=150 \mathrm{~K}$, the potential energy quickly decreases during the first 200 ps before stabilizing, which confirms the grain- and interphase boundaries relaxation. Non-periodic shrink-wrapped boundary conditions are used, as well as a timestep of 1 fs. Finally, the nanoparticle is quenched down to a temperature of $5 \mathrm{~K}$ at which the nanocompression simulation is performed.

\section{Nanocompression MD simulation}

The MD nanocompression setup has been described previously. ${ }^{44,45}$ In brief, each nanoparticle is placed between two (top and bottom) infinite and planar force fields (force axis-aligned with $<111>$ ). The top indenter is moved at constant displacement rate equivalent to an engineering strain rate of $10^{8} \mathrm{~s}^{-1}$ while the substrate is kept fixed. While the MD simulations of deformation are performed at strain rates which are much higher than in the experiment, they provide good estimates for stress distribution in the particles and help to identify the atomistic deformation mechanisms. The load and displacement of the indenter are recorded on-the-fly during the simulation. Local crystal structures and defects are characterized using Ovito including DXA, displacement vectors and polyhedral template matching analysis. ${ }^{46,47}$

\section{Supporting Information}

The Supporting Information is available free of charge via the Internet at: http://pubs.acs.org

- Energy dispersive X-ray spectroscopy map of C, O, Ag, Au, and Pt distribution in the $\mathrm{Au}$ shell of as-fabricated Ag-Au core-shell nanoparticle (Figure S1), Load-displacement curves of single crystalline Ag nanoparticles (Figure S2).

- An estimate (based on Fick's second law) of diffusion length of Ag atoms along the grain boundaries in the Au shell at room temperature.

- Contact model of flat rigid punch with the polycrystalline Au shell and the derivation of the expression for the mean contact pressure when a full contact 
of the flat punch with a polycrystalline shell is established, the derivation being supported by Fig. S3.

- The analysis of transferability of embedded atom method (EAM) Ag-Au interatomic potential with the comparison of calculated and experimental material properties for Ag (Table S1) and Au (Table S2).

- Movie M1: the MD simulations of the dislocation nucleation events during compression of core-shell nanoparticle; the middle cross-section of the particle being shown.

\section{ACKNOWLEDGEMENTS}

This work was supported by the Israel Science Foundation, grant No. 617/19, by the Russell Berrie Nanotechnology Institute at the Technion, and by the French ANR (project SASHA), grant No. ANR-20-CE09-0015.

Author contributions: AS has performed the experiments, with participation of NG and YQ. JA has performed the atomistic simulations. NG has fabricated the samples. ER has developed the idea. ER and OT have secured the funding. ER and JA have written the manuscript, with contributions from all co-authors.

Competing interests: All authors declare no competing interests.

\section{REFERENCES}

(1) Richter, G.; Hillerich, K.; Gianola, D. S.; Mönig, R.; Kraft, O.; Volkert, C. A. Ultrahigh Strength Single Crystalline Nanowhiskers Grown by Physical Vapor Deposition. Nano Lett. 2009, 9 (8), 3048-3052.

https://doi.org/10.1021/n19015107.

(2) Chen, L. Y.; He, M. R.; Shin, J.; Richter, G.; Gianola, D. S. Measuring Surface Dislocation Nucleation in Defect-Scarce Nanostructures. Nat. Mater. 2015, 14 (7), 707-713. https://doi.org/10.1038/nmat4288.

(3) Mordehai, D.; Lee, S. W.; Backes, B.; Srolovitz, D. J.; Nix, W. D.; Rabkin, E. Size Effect in Compression of Single-Crystal Gold Microparticles. Acta Mater. 2011, 59 (13), 5202-5215. https://doi.org/10.1016/j.actamat.2011.04.057.

(4) Sharma, A.; Hickman, J.; Gazit, N.; Rabkin, E.; Mishin, Y. Nickel Nanoparticles Set a New Record of Strength. Nat. Commun. 2018, 9 (1), 4102(1-9). https://doi.org/10.1038/s41467-018-06575-6.

(5) Sharma, A.; Kositski, R.; Kovalenko, O.; Mordehai, D.; Rabkin, E. Giant Shape- and Size-Dependent Compressive Strength of Molybdenum Nano- and Microparticles. Acta Mater. 2020, 198, 72-84. https://doi.org/10.1016/j.actamat.2020.07.054.

(6) Chatain, D.; Ghetta, V.; Wynblatt, P. Equilibrium Shape of Copper Crystals Grown on Sapphire. Interface Sci. 2004, 12 (1), 7-18. https://doi.org/10.1023/B:INTS.0000012290.07441.a8.

(7) Gerberich, W. W.; Nelson, J. C.; Lilleodden, E. T.; Anderson, P.; Wyrobek, J. 
T. Indentation Induced Dislocation Nucleation: The Initial Yield Point. Acta Mater. 1996, 44 (9), 3585-3598. https://doi.org/10.1016/1359-6454(96)000109.

(8) Corcoran, S.; Colton, R.; Lilleodden, E.; Gerberich, W. Anomalous Plastic Deformation at Surfaces: Nanoindentation of Gold Single Crystals. Phys. Rev. B - Condens. Matter Mater. Phys. 1997, 55 (24), R16057-R16060. https://doi.org/10.1103/PhysRevB.55.R16057.

(9) Sharma, A.; Gazit, N.; Klinger, L.; Rabkin, E. Pseudoelasticity of Metal Nanoparticles Is Caused by Their Ultrahigh Strength. Adv. Funct. Mater. 2020, 30 (18), 1-8. https://doi.org/10.1002/adfm.201807554.

(10) Greer, J. R.; Nix, W. D. Nanoscale Gold Pillars Strengthened through Dislocation Starvation. Phys. Rev. B - Condens. Matter Mater. Phys. 2006, 73 (24), 1-6. https://doi.org/10.1103/PhysRevB.73.245410.

(11) Lee, S. W.; Mordehai, D.; Rabkin, E.; Nix, W. D. Effects of Focused-Ion-Beam Irradiation and Prestraining on the Mechanical Properties of FCC Au Microparticles on a Sapphire Substrate. J. Mater. Res. 2011, 26 (14), 16531661. https://doi.org/10.1557/jmr.2011.221.

(12) Csikor, F. F.; Motz, C.; Weygand, D.; Zaiser, M.; Zapperi, S. Dislocation Avalanches, Strain Bursts, and the Problem of Plastic Forming at the Micrometer Scale. Science (80-. ). 2007, 318 (5848), 251-254. https://doi.org/10.1126/science.1143719.

(13) Van Swygenhoven, H.; Derlet, P. M.; Hasnaoui, A. Atomic Mechanism for Dislocation Emission from Nanosized Grain Boundaries. Phys. Rev. B Condens. Matter Mater. Phys. 2002, 66 (2), 024101(1-8). https://doi.org/10.1103/PhysRevB.66.024101.

(14) Gu, X. W.; Loynachan, C. N.; Wu, Z.; Zhang, Y. W.; Srolovitz, D. J.; Greer, J. R. Size-Dependent Deformation of Nanocrystalline Pt Nanopillars. Nano Lett. 2012, 12 (12), 6385-6392. https://doi.org/10.1021/n13036993.

(15) Van Swygenhoven, H.; Derlet, P. M.; Frøseth, A. G. Stacking Fault Energies and Slip in Nanocrystalline Metals. Nat. Mater. 2004, 3 (6), 399-403. https://doi.org/10.1038/nmat1136.

(16) Hyde, B.; Espinosa, H. D.; Farkas, D. An Atomistic Investigation of Elastic and Plastic Properties of Au Nanowires. Jom 2005, 57 (9), 62-66. https://doi.org/10.1007/s11837-005-0118-x.

(17) Jang, D.; Cai, C.; Greer, J. R. Influence of Homogeneous Interfaces on the Strength of $500 \mathrm{~nm}$ Diameter Cu Nanopillars. Nano Lett. 2011, 11 (4), 17431746. https://doi.org/10.1021/nl2003076.

(18) Afanasyev, K. A.; Sansoz, F. Strengthening in Gold Nanopillars with Nanoscale Twins. Nano Lett. 2007, 7 (7), 2056-2062. https://doi.org/10.1021/n10709591.

(19) Li, Y. P.; Zhang, G. P.; Wang, W.; Tan, J.; Zhu, S. J. On Interface Strengthening Ability in Metallic Multilayers. Scr. Mater. 2007, 57 (2), 117120. https://doi.org/10.1016/j.scriptamat.2007.03.032.

(20) Raghavan, R.; Wheeler, J. M.; Harzer, T. P.; Chawla, V.; Djaziri, S.; Thomas, 
K.; Philippi, B.; Kirchlechner, C.; Jaya, B. N.; Wehrs, J.; Michler, J.; Dehm, G. Transition from Shear to Stress-Assisted Diffusion of Copper-Chromium Nanolayered Thin Films at Elevated Temperatures. Acta Mater. 2015, 100, 7380. https://doi.org/10.1016/j.actamat.2015.08.016.

(21) Sun, S.; Kong, D.; Li, D.; Liao, X.; Liu, D.; Mao, S.; Zhang, Z.; Wang, L.; Han, X. Atomistic Mechanism of Stress-Induced Combined Slip and Diffusion in Sub-5 Nanometer-Sized Ag Nanowires. ACS Nano 2019, 13 (8), 8708-8716. https://doi.org/10.1021/acsnano.9b00474.

(22) Sun, J.; He, L.; Lo, Y.-C.; Xu, T.; Bi, H.; Sun, L.; Zhang, Z.; Mao, S. X.; Li, J. Liquid-like Pseudoelasticity of sub-10-nm Crystalline Silver Particles. Nat. Mater. 2014, 13 (11), 1007-1012. https://doi.org/10.1038/nmat4105.

(23) Ng, K. S.; Ngan, A. H. W. Deformation of Micron-Sized Aluminium BiCrystal Pillars. Philos. Mag. 2009, 89 (33), 3013-3026. https://doi.org/10.1080/14786430903164614.

(24) Kunz, A.; Pathak, S.; Greer, J. R. Size Effects in Al Nanopillars: Single Crystalline vs. Bicrystalline. Acta Mater. 2011, 59 (11), 4416-4424. https://doi.org/10.1016/j.actamat.2011.03.065.

(25) Imrich, P. J.; Kirchlechner, C.; Motz, C.; Dehm, G. Differences in Deformation Behavior of Bicrystalline $\mathrm{Cu}$ Micropillars Containing a Twin Boundary or a Large-Angle Grain Boundary. Acta Mater. 2014, 73, 240-250. https://doi.org/10.1016/j.actamat.2014.04.022.

(26) Kheradmand, N.; Vehoff, H.; Barnoush, A. An Insight into the Role of the Grain Boundary in Plastic Deformation by Means of a Bicrystalline Pillar Compression Test and Atomistic Simulation. Acta Mater. 2013, 61 (19), 74547465. https://doi.org/10.1016/j.actamat.2013.08.056.

(27) Heller, M.; Gibson, J. S. K. L.; Pei, R.; Korte-Kerzel, S. Deformation of $\mu \mathrm{m}-$ and $\mathrm{mm}$-Sized Fe2.4wt\%Si Single- and Bi-Crystals with a High Angle Grain Boundary at Room Temperature. Acta Mater. 2020, 194, 452-463. https://doi.org/10.1016/j.actamat.2020.04.011.

(28) Qin, Q.; Yin, S.; Cheng, G.; Li, X.; Chang, T. H.; Richter, G.; Zhu, Y.; Gao, H. Recoverable Plasticity in Penta-Twinned Metallic Nanowires Governed by Dislocation Nucleation and Retraction. Nat. Commun. 2015, 6 (1), 1-8. https://doi.org/10.1038/ncomms6983.

(29) Gazit, N.; Klinger, L.; Richter, G.; Rabkin, E. Formation of Hollow GoldSilver Nanoparticles through the Surface Diffusion Induced Bulk Intermixing. Acta Mater. 2016, 117, 188-196. https://doi.org/10.1016/j.actamat.2016.07.009.

(30) Bahr, D. F.; Kramer, D. E.; Gerberich, W. W. Non-Linear Deformation Mechanisms during Nanoindentation. Acta Mater. 1998, 46 (10), 3605-3617. https://doi.org/10.1016/S1359-6454(98)00024-X.

(31) Haag, S. T.; Richard, M. I.; Welzel, U.; Favre-Nicolin, V.; Balmes, O.; Richter, G.; Mittemeijer, E. J.; Thomas, O. Concentration and Strain Fields inside a $\mathrm{Ag} / \mathrm{Au}$ Core-Shell Nanowire Studied by Coherent X-Ray Diffraction. Nano Lett. 2013, 13 (5), 1883-1889. https://doi.org/10.1021/nl303206u. 
(32) Volmer, M.; Weber, A. Keimbildung in Übersättigten Gebilden. Zeitschrift für Phys. Chemie 1926, $119 U$ (1), 277-301. https://doi.org/10.1515/zpch-192611927.

(33) Minkwitz, C.; Herzig, C.; Rabkin, E.; Gust, W. The Inclination Dependence of Gold Tracer Diffusion along a $\Sigma 3$ Twin Grain Boundary in Copper. Acta Mater. 1999, 47 (4), 1231-1239. https://doi.org/10.1016/S13596454(98)00429-7.

(34) Mullins, W. W. Theory of Thermal Grooving. J. Appl. Phys. 1957, 28 (3), 333339. https://doi.org/10.1063/1.1722742.

(35) Martin, J. W.; Doherty, R. D.; Cantor, B. Stability of Microstructure in Metallic Systems; Cambridge University Press, 1997. https://doi.org/10.1017/CBO9780511623134.

(36) Issa, I.; Amodeo, J.; Réthoré, J.; Joly-Pottuz, L.; Esnouf, C.; Morthomas, J.; Perez, M.; Chevalier, J.; Masenelli-Varlot, K. In Situ Investigation of MgO Nanocube Deformation at Room Temperature. Acta Mater. 2015, 86 (C), 295304. https://doi.org/10.1016/j.actamat.2014.12.001.

(37) Hirel, P. Atomsk: A Tool for Manipulating and Converting Atomic Data Files. Comput. Phys. Commun. 2015, 197, 212-219.

https://doi.org/10.1016/j.cpc.2015.07.012.

(38) Plimpton, S. Fast Parallel Algorithms for Short-Range Molecular-Dynamics. $J$. Comput. Phys. 1995, 117 (1), 1-19. https://doi.org/10.1006/jcph.1995.1039.

(39) Zhou, X. W.; Johnson, R. A.; Wadley, H. N. G. Misfit-Energy-Increasing Dislocations in Vapor-Deposited CoFe/NiFe Multilayers. Phys. Rev. B 2004, 69 (14), 1358-10. https://doi.org/10.1103/physrevb.69.144113.

(40) Zhou, X. W.; Wadley, H. N. G.; Johnson, R. A.; Larson, D. J.; Tabat, N.; Cerezo, A.; Petford-Long, A. K.; Smith, G. D. W.; Clifton, P. H.; Martens, R. L.; Kelly, T. F. Atomic Scale Structure of Sputtered Metal Multilayers. Acta Mater. 2001, 49 (19), 4005-4015. https://doi.org/10.1016/s13596454(01)00287-7.

(41) Pereira, Z. S.; Silva, E. Z. da. Cold Welding of Gold and Silver Nanowires: A Molecular Dynamics Study. J. Phys. Chem. C 2011, 115 (46), 22870-22876. https://doi.org/10.1021/jp207842v.

(42) Moseley, P.; Curtin, W. A. Computational Design of Strain in Core-Shell Nanoparticles for Optimizing Catalytic Activity. Nano Lett. 2015, 15 (6), 4089-4095. https://doi.org/10.1021/acs.nanolett.5b01154.

(43) Bitzek, E.; Koskinen, P.; Gähler, F.; Moseler, M. Structural Relaxation Made Simple. Phys. Rev. Lett. 2006, 97, 170201. https://doi.org/10.1103/physrevlett.97.170201.

(44) Amodeo, J.; Lizoul, K. Mechanical Properties and Dislocation Nucleation in Nanocrystals with Blunt Edges. Mater. Des. 2017, 135, 223-231. https://doi.org/10.1016/j.matdes.2017.09.009.

(45) Goryaeva, A. M.; Fusco, C.; Bugnet, M.; Amodeo, J. Influence of an Amorphous Surface Layer on the Mechanical Properties of Metallic Nanoparticles under Compression. Phys. Rev. Mater. 2019, 3 (3), 33606. 
https://doi.org/10.1103/physrevmaterials.3.033606.

(46) Stukowski, A. Visualization and Analysis of Atomistic Simulation Data with OVITO-the Open Visualization Tool. Model. Simul. Mater. Sci. Eng. 2010, 18 (1), 7pp. https://doi.org/10.1088/0965-0393/18/1/015012.

(47) Larsen, P. M.; Schmidt, S.; Schiøtz, J. Robust Structural Identification via Polyhedral Template Matching. Model. Simul. Mater. Sci. Eng. 2016, 24 (5), 055007-055019. https://doi.org/10.1088/0965-0393/24/5/055007. 